\title{
Variable Refrigerant Flow Systems on USACE Projects
}

\author{
Scott W. Kramer, Ph.D.*, Matthew Kilmer, Keith Rahn \\ Auburn University, Auburn, Alabama, 36849, USA
}

\begin{abstract}
Variable Refrigerant Flow (VRF) systems offer a unique set of advantages and disadvantages to a designer, contractor, and building owner. With the procurement requirements for the Government, additional challenges are introduced by these systems, and the systems are currently not permitted for US Army Corps of Engineers (USACE) projects due to requirements in mandatory design criteria. This research was performed to discover the major advantages and disadvantages of these systems, and to understand why they are not allowed on USACE projects. While performing interviews concerning these types of systems, two interesting case studies that demonstrate some of the disadvantages for these systems were discovered and will be detailed in this paper. Ultimately, it was discovered that VRF systems do not meet the public law requirement to provide open protocol control systems for U.S. Military projects. While the reasoning for this research had a particular focus on U.S. Military construction, much of the information presented will also be valuable to private entities considering these systems as well.
\end{abstract}

C 2019 The Authors. Published by Budapest University of Technology and Economics \& Diamond Congress Ltd. Peer-review under responsibility of the scientific committee of the Creative Construction Conference 2019.

Keywords: VRF, Variable Refrigerant Flow, Inverter Compressor, Efficiency;

\section{Introduction}

VRF systems are a relatively new type of HVAC systems used in America. These systems take advantage of using a variable capacity compressor to provide a higher efficiency than can be achieved using the more standard types of compressors that have historically been used in HVAC systems like the standard scroll or reciprocating compressor. Less power is consumed by the VRF system at part load compared to conventional systems, which is due to the variable speed driven compressors and fans at outdoor sections. [1]

The potential use of VRF systems has gone through three iterations within design criteria for US Army Corps of Engineers' (USACE) projects. The three iterations will be discussed in more detail below. The current design criteria would allow the use of this technology, but there are no vendors that meet all of the prescriptive requirements, so the use of these types of systems are still essentially not allowed. VRF systems have a variety of advantages and disadvantages that will be explored in detail in this paper. Advantages range from energy efficiency to potentially being able to reduce the required height of a building due to the principles behind using these types of systems. Disadvantages range from concerns over parts and service availability due to the proprietary nature of these systems to safety concerns due to the amount of refrigerant that will be in the piping in the occupied portion of the building.

*kramesw@auburn.edu 
Scott W. Kramer, et al. / Proceedings of the Creative Construction Conference (2019) 022 https://doi.org/10.3311/CCC2019-022

Much of the literature for these types of systems suggest that they are among the most efficient systems available for use in the HVAC market today. So, this research was performed to provide an evaluation between the advantages and disadvantages of these types of systems with a particular focus on what benefits may be lost since the military design criteria essentially disallows these systems.

While performing interviews for these types of systems, two interesting case studies that demonstrate some of the disadvantages for these systems were discovered from discussions with maintenance and USACE staff. These case studies support the concerns raised in the USACE design criteria that do not allow these systems. The significant problem associated with these systems for military projects is the need for very thorough training for both installing contractors and maintenance staff.

\section{Background}

Selecting the type of HVAC system is obviously a critical decision that must be made early in the life of a project. This decision will be one of the primary factors that determines a large portion of the utility cost for the life of the building. Selecting an efficient type of system is critical as the portion of energy use in buildings that is used inefficiently or unnecessarily is 30 percent. [2] For smaller projects, options are limited due to the types of equipment that are manufactured to handle smaller loads and typically a refrigerant based packaged unit or split system will be selected. Larger projects have a wide variety of options available including constant volume systems, variable air volume systems, boilers, chillers, packaged/rooftop units, water source heat pumps, and one of the newer technologies available is Variable Refrigerant Volume (VRF) systems. While VRF systems are relatively new in the United States, they have been in use in other countries for longer durations. Variable refrigerant flow systems have been used in Japan for at least two decades and are now receiving attention in North America as a potential HVAC system choice in commercial, retail, institutional, hospitality, and multifamily residential applications. [3] One of the main reasons that a building owner may wish to use a VRF system is the flexibility that this system has to adapt to the building load. The main advantage of a variable refrigerant flow (VRF) system is its ability to respond to fluctuations in space load conditions. By comparison, conventional direct expansion (DX) systems offer limited or no modulation in response to changes in the space load conditions. [1] In other words, a VRF system can handle less than peak conditions in a building and still provide the building user with comfort which is a problem for traditional DX systems due to the inability of the system to run at a reduced capacity. Other advantages of these systems will be discussed in more detail later in this paper.

\section{Purpose \& Rational for the Study}

As VRF systems are not currently allowed to be used for US Army Corps of Engineers' projects, there is not as much history and knowledge within the Corps of Engineers for these systems as there is for other types of systems. The purpose of this study was to explain to the reader:

- How VRF technology works.

- The options available when using VRF systems.

- The reasons that the US Army Corps of Engineers design guidance does not allow VRF systems

- The advantages and disadvantages that need to be considered when using VRF systems.

Space heating, cooling, and ventilation costs make up approximately $33 \%$ of the energy costs for commercial buildings as can be seen in Figure 1 below. [4] So, the need to find energy efficient solutions to minimize operating costs is a constant dynamic for building owners, including the Government. However, the US Army Corps of Engineers' current design guidance all but eliminates the possibility of using VRF systems, a potentially efficient system, based on the numerous strict requirements that currently no VRF vendor meets. As the rest of the commercial market seems to be shifting towards accepting VRF, it was a question as to why their use was disallowed. The VRF market was valued at $\$ 11.08$ billion in 2015 and is expected to reach $\$ 24.09$ billion by 2022. [5] With the use of VRF increasing in the 
Scott W. Kramer, et al. / Proceedings of the Creative Construction Conference (2019) 022 https://doi.org/10.3311/CCC2019-022

private world, this study was performed to identify the reasons that the Corps of Engineers does not allow these systems and what potential advantages may be lost by not using these systems. This study resulted in a series of items, both good and bad, to consider when potentially considering the use of VRF systems for a project.

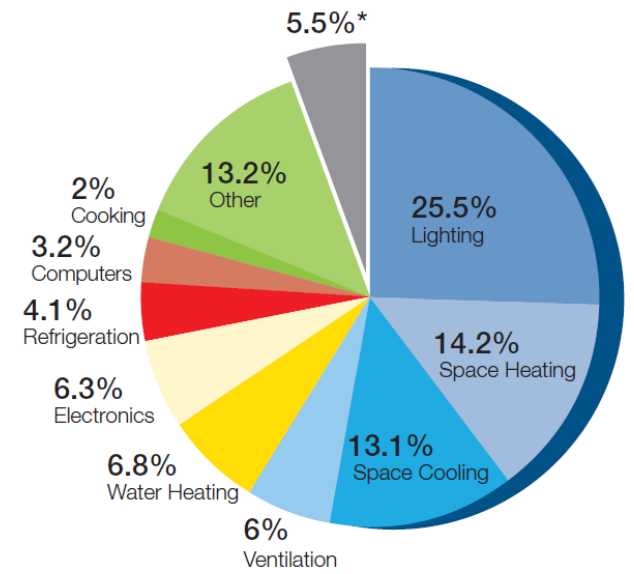

Figure 1: Commercial Primary End Use (US Department of Energy, 2008)

\section{Research Design}

This study evaluated various publications and vendor-based literature to provide the reader with a basic understanding of the VRF systems and the options available when using these types of systems. This study also used various literature and interviews obtained from a wide variety of industry professionals to obtain data for these systems including private design engineers, commissioning agents, maintenance staff, installing contractors, and various US Army Corps of Engineers' personnel to evaluate the advantages and disadvantages that have been experienced while designing, installing, and maintaining these systems. Finally, this study also used interviews from US Army Corps of Engineers' personnel to gain a deeper insight as to why the use of these systems has been highly discouraged within the past and current design guidance. This study used qualitative research methods to provide the reader with the desired data as explained in the purpose of this study.

\section{Data Analysis}

Variable Refrigerant Volume (VRV) systems were invented in 1982 by Daikin. The company trademarked the name VRV, resulting in all other manufacturers calling their product VRF. [6] There are approximately ten vendors that are competitors in the VRF market in America based on searching the internet for companies that offer these types of systems. There are significantly more companies providing these systems in other countries. A traditional refrigerant based air conditioning or heat pump system consists of one outdoor unit containing a compressor coupled with one indoor air handling unit and the units are either on or off based on a call for cooling or heating from a thermostat. A VRF system is very different from a standard refrigerant based split system in that one outdoor unit can be connected to multiple indoor units. Another substantial difference from an efficiency and comfort standpoint is that both the indoor and outdoor units in a VRF system can operate at variable capacities as the load in each zone changes.

\subsection{Components of VRF Systems}

VRF systems consist of an outdoor unit with a variable capacity compressor, various types of indoor units with variable speed fans, and depending on the type of system and unit manufacturer, some type of heat recovery control unit may also be present. A heat recovery control unit is essentially a box with multiple refrigerant line connections that controls the flow of refrigerant throughout the system. Data below came from reviewing literature from the following VRF manufacturer product references. [7] [8] [9] [10] 
One common feature of VRF systems is there are multiple capacities available for the outdoor units. Based on reviewing various vendors' available options, this range is from as small as a 2-ton unit to as large as a 42-ton unit for a single outdoor unit. Also, multiple outdoor units can be manifolded together to make a larger system. Each manufacturer has a different limit for the number of units that can be combined, but it is typically 2-4 outdoor units.

Each of these outdoor units can be coupled to multiple indoor units. Indoor unit capacities can range from 0.5 tons to 8 tons in various configurations. Vendors have different limits for how many indoor units can be coupled together. The range was from 1 indoor unit up to typically 40-50 indoor units, but one vendor allowed up to 64 indoor units per outdoor unit. The indoor units are available in a wide variety of configurations that allows an engineer to select an appropriate unit for the type of space it is serving. Some examples of the ductless options available are wall mounted, ceiling mounted, and floor standing. There are also horizontal and vertical ducted options available. Images of a few of these typical units are depicted below in Figure 2.
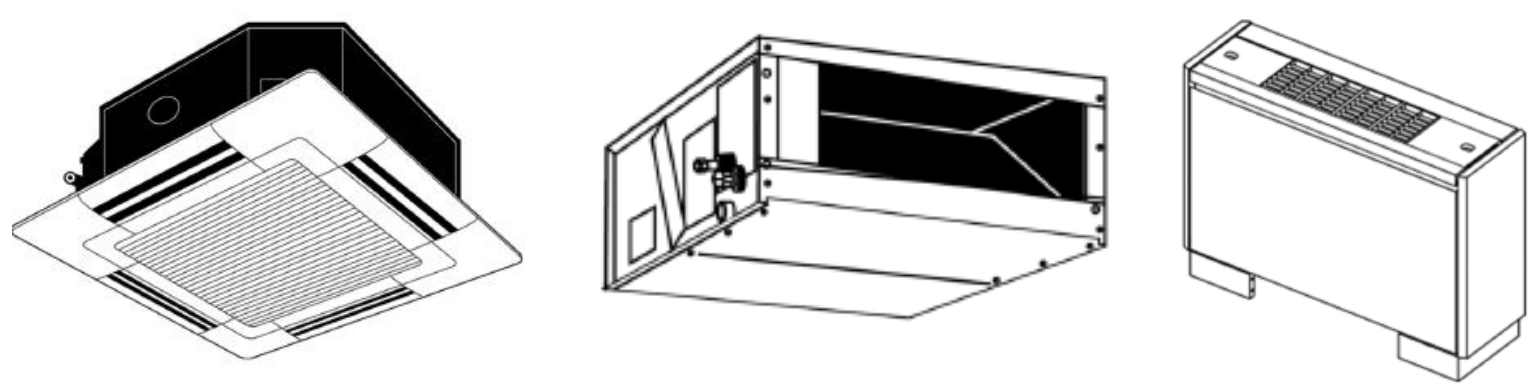

Figure 2: Indoor VRF Units: Ceiling Cassette, Concealed Ducted, Floor-Standing Exposed [11]

A final typical component is the heat recovery control unit. Each manufacturer has a branded trade name for this unit, but the operation of the unit is relatively consistent. This unit is a box with multiple refrigerant piping connections that distributes refrigerant throughout the piping network, and it is the piece that would allow refrigerant to be cycled between units that are simultaneously operating in heating and cooling without mechanical compression. This is a selling point of VRF systems in that if you have a building that has a need for both heating and cooling at the same time, it can be accomplished in a very efficient manner using this device.

\subsection{Types of VRF Systems}

VRF systems are available in three types of operational configurations: cooling only, heat pump, and heat recovery. Each of these arrangements has a different amount of hardware and software required for the application. The detailed design rationale for using each type of system is outside the scope of this paper, and only a basic introduction to each type of available configuration will be provided. First, the cooling only arrangement requires that all attached units be in the same operational mode and there is no heating provided by this type of arrangement. Heating would need to be provided from an additional source like potentially perimeter baseboard heaters if using this configuration. This type of system would be a potential benefit for spaces that have a constant supply of heat and no demand for additional heating like a computer room or telecommunications room. Second, a heat pump arrangement also requires that each interior unit be in the same operational mode, but this type of system has the added benefit that the entire system can be in the heating mode and the space can be heated from the VRF system versus needing a separate system for heating. This type of system would be a potential benefit for a space that has a uniform heating and cooling load throughout with no need for different operational modes in different parts of the space. The third and most advanced arrangement that utilizes the full capability of this technology is the heat recovery arrangement. Heat recovery systems are heat pump systems that can provide simultaneous heating and cooling within the same system. All indoor units connected to a heat recovery system can not only use individual control and setpoints, but they can also individually operate in heating or cooling mode at any given time. Manufacturers offer two design approaches to VRF heat recovery operation: two-pipe and three-pipe systems. [11] Knowledge of the various piping arrangements and piping connections to be used are critical to consider from a design and installation stand point and will be discussed further below under the disadvantages of these systems. 
Scott W. Kramer, et al. / Proceedings of the Creative Construction Conference (2019) 022 https://doi.org/10.3311/CCC2019-022

\subsection{US Army Corps of Engineers Design Guidance for VRF Systems}

The design and construction criteria for the Corps of Engineers is very structured. The overall design guidance for facilities is found in various documents called Unified Facilities Criteria (UFC). This type of criteria is similar to model building codes used in the private/commercial sector in that they provide higher level criteria typically focused on how the building should be designed. The more prescriptive construction specifications are found in documents called Unified Facilities Guide Specifications (UFGS). These specifications are similar to other construction specifications used in the private/commercial sector but are in general more stringent that standard commercial specifications. The information discussed below is summarized from researching archived versions of UFGS specifications and UFC design criteria.

Initially, VRF systems were not specifically mentioned in either the UFC or the UFGS documents. As the technology was relatively new, the criteria had not been updated to reflect this new type of system. There was some informal guidance circulated that pointed out that VRF systems do not meet the open protocol requirements that were found within the UFGS specification 230923 for Direct Digital Control for HVAC systems. This specification was in place from approximately 2006 until 2015 when it was replaced with a very similarly worded specification. These specifications required that all equipment communicating on a control system communicate over an open (nonproprietary) communication protocol. Based on reviewing manufacturer's literature and interviews with an industry expert in this subject, there are no VRF vendors that use a non-proprietary control system. However, this information was either not well circulated, possibly waived on a project by project basis, or ignored as there have been many buildings on Army and Air Force installations provided with VRF systems since that time.

In January 2017, the UFC design guidance was updated to specifically address VRF systems. UFC 3-410-01, Heating, Ventilating, and Air Conditioning Systems, included language specifically for these systems. This was the first time that VRF systems were formally addressed in the design criteria. The language used specifically disallowed these systems to be used on Air Force projects, but the language was extremely vague for Army projects. The language that was used was: "For Army facilities, VRF systems are strongly discouraged". While the intent that the systems were not desired was clear, from a design or construction enforcement standpoint, the language was not enforceable as the use of the words "discouraged" is not contractually enforceable, so ultimately each project team and authority having jurisdiction could choose to use these systems on Army projects. The next, and current, iteration of design guidance for VRF systems cleared up the ambiguous language from the previous guidance. UFC 3-410-01 was revised in November of 2017. This guidance contains a list of nine prescriptive requirements that must be met if VRF systems are going to be used. One of these requirements is the use of an open protocol control system as discussed above. The UFC actually states that as of the publication date all known commercially-available VRF systems rely on a proprietary network. Another restrictive requirement is the use of only brazed piping connections. Based on my review of manufacturers' data, there are many of these VRF components that come standard with flared piping connections. So, while the current guidance theoretically would allow VRF systems to be used, realistically no vendor can meet these requirements. Another major concern discussed in the UFC is the amount of refrigerant that will be ran through the occupied portion of the building when using these systems that can potentially leak into the building.

As a part of this research, an interview with a USACE member that played a large part in developing these design criteria was conducted. He personally felt that the systems had great advantages and hoped that industry will adjust to meet the Government's prescriptive requirements over time. His explanation for the prescriptive requirements was that the requirement for an open protocol control system is not just a matter of preference, it is actually a public law. Title 10 of the United States Code Section 2867 requires open protocol control systems in military construction. He further explained that he felt this was an unfortunate disconnect between Government and industry stating that the Government wants full and complete ownership of our systems, and industry wants to protect their proprietary elements that drive their technology and thus their business. This same sentiment was reflected in another interview with a senior USACE mechanical engineer by stating that VRF is a tool that you don't want to take off the table, but the Corps doesn't know how to deal with it yet.

\subsection{Advantages of VRF Systems}

Based on interviews with a variety of HVAC professionals and researching various publications for VRF systems, the perceived advantages mesh well with the advantages that are published. The items below were cited as advantages during interviews by more than one interviewee. 
Scott W. Kramer, et al. / Proceedings of the Creative Construction Conference (2019) 022 https://doi.org/10.3311/CCC2019-022

The most cited advantage was that these systems work very well for buildings with limited ceiling space. This element provides a huge benefit for the renovation of older buildings where space is often limited. The main foot print of the VRF mechanical system is made up of smaller refrigerant lines instead of larger ductwork. VRF systems require much less ceiling space than conventional systems because only the refrigerant piping and ducted outside ventilation air are accommodated. [12] As larger ductwork is not needed with this type of system to provide space conditioning, less ceiling space is needed, so there is the potential to reduce the height of a building which can save a substantial amount of money. Another benefit was that life cycle cost of VRF systems can often be lower as higher efficiencies than other systems may be achieved depending on the use of the building. Especially if a building allows for the heat recovery (simultaneous heating and cooling) feature to be used, this system will be extremely efficient. Potentially up to a 58\% savings can be realized on energy costs [13] Occupancy satisfaction has a better chance of success due to better environmental controls and each office generally has a dedicated thermostat when using this type of system. Better environmental controls are provided from the variable capacity elements of this system versus other systems that may only have staged operations versus fully modulating operations. The system adjusts the flow of refrigerant to each indoor unit based on its operating conditions. It computes the amount of refrigerant required by each indoor unit and controls the refrigerant flow to ensure desired comfort level without over cooling or heating of the space. [14]

\subsection{Disadvantages of VRF Systems}

The most cited disadvantage was the need for very specific, detailed manufacturer based training for not only the long term maintenance of these systems, but also the initial installation of these systems. As previously mentioned, every vendor has their own proprietary control system that must be learned. Every manufacturer has their own unique way of executing this technology, and it is critical that the staff installing and maintain these systems receive extensive training. Training becomes even more crucial when you factor in that most HVACR manufacturers will not grant contractors permission to stock their systems, nor honour equipment warranties, unless installing contractors are properly trained and hold the necessary certifications. [15] Finding qualified or certified maintenance personnel must be a consideration when considering using these systems. Local parts availability must also be considered. A case study demonstrating the critical nature of this disadvantage is provided below.

The proprietary controls included in these systems is a major disadvantage as detailed above, but it is not just the controls that are proprietary in these systems. Each vendor has other proprietary components and the major components from one vendor are not compatible with other vendors. This element complicates both the design and procurement of these types of systems difficult as the Government cannot sole source the design to one particular vendor due to full and open procurement requirements. Designing a VRF system for competitive bid is challenging due to each manufacturer's unique implementation of the VRF system technology. Each has its own set of requirements and restrictions that must be considered. However, if the system is designed solely for a particular manufacturer's requirements, the design will be hard to bid by others. In particular, the capabilities of the proprietary central controls systems are vastly different. [16] One interviewee also expressed that the proprietary challenges of these systems can make it difficult to integrate these systems to a central monitoring system and to develop good test procedures

The risk of a large refrigerant leak is also a disadvantage. According to ASHRAE Standard 15, a VRF system is classified as a direct system/high-probability system where a refrigerant leak can potentially enter into the occupied space. [12] Standard 15, Safety Standard for Refrigerant Systems, strives to ensure a safe system by limiting the maximum quantity of refrigerant below that which is a danger to human occupants if a leak occurs. Manufacturers of VRF systems components advertise that 40 or more evaporators can be included on one piping network with more than $3,000 \mathrm{ft}$. (914 m) of refrigerant pipe. A traditional DX split system applied room-by-room has one condensing unit for each evaporator, with no interconnection to other split systems, and a refrigerant leak, therefore would discharge only that refrigerant contained in one individual split system. Because of the interconnecting refrigerant piping, a VRF system has the theoretical potential to discharge a much larger quantity of refrigerant to indoor spaces in a catastrophic leak occurrence. [3] UFC 3-410-01 further restricts the total refrigerant charge to 49 pounds or less.

Related to the refrigerant leak disadvantage is that with the large amount of refrigerant piping in the building, there will be a corresponding large number of refrigerant joints. Finding refrigerant leaks is much more difficult than finding water leaks as there is no visible evidence of the leak from a refrigerant leak as there is with water. Two interviewees had personally experienced major problems with leaks during the installation of VRF systems. One of these examples 
Scott W. Kramer, et al. / Proceedings of the Creative Construction Conference (2019) 022 https://doi.org/10.3311/CCC2019-022

is provided as a case study below. Fortunately, these leaks occurred during construction, but if a leak occurs after a building is occupied, it could be a disaster as it will be even more difficult to find a leak once the building is full of people and furniture. A disadvantage unique to the Government is that many of these systems are manufacturers in foreign countries that can introduce a challenge to the Buy American procurement requirements for Government construction contracts. Another consideration for VRF systems is that a separate ventilation system must be provided as these systems are typically not ducted nor do they provide the capacity to handle the heavy latent load that can come with ventilation air for various parts of the world. Currently, ventilation systems used in conjunction with VRF systems are engineered on a case-by-case basis. Manufacturers are evaluating potential approaches for an integrated solution. [17]

\subsection{VRF System Failure at Ft. Stewart, Georgia, USA}

Ft. Stewart hired a contractor to build a child care center. This type of facility functions very similar to a "day care" facility in the private world but it can potentially be open 24 hours a day. A VRF system was installed in this building by a mechanical contractor that was not a local contractor. This contractor provided a VRF system that met all of the performance criteria in the contract, but they used one of the less prevalent VRF systems in the local market. The installation's maintenance staff were provided with minimal training for this system when the project was completed and turned over. As luck would have it, approximately two months after the one-year construction warranty had expired, the VRF system had a failure that resulted in the entire system being non-operational. The installation's maintenance staff attempted to diagnose the problem, but had not been provided adequate training, or the vendor's necessary proprietary software to allow them to further troubleshoot. The building had to be closed temporarily as they could not get the system back running. The building sat vacant for a while and ultimately was converted to be used for office space. The installation's maintenance staff reached out to all of the local service organizations but could not find anyone that was certified to work on this particular vendor's system. The maintenance staff ended up installing window air conditioning units in this building so that it could actually be used. During this time, one of the local service contractors received the proper factory training and software and was able to get the system running again. This case study clearly demonstrates one of the major drawbacks for VRF which is the need for specialized training for maintenance staff for these systems. Again, it is critical to have knowledge of not only the mechanical components of these systems, but also the proprietary control system and the associated software. Had adequate training and software been provided this situation could have been avoided.

\subsection{VRF System Installation Difficulties at Ft. Benning, Georgia, USA}

Ft. Benning had the need to convert a very old barracks building with very limited ceiling height into an office building. With the limited ceiling height, a VRF system was a very good option as there was not room for a large central duct system in the building and floor space was also extremely limited. The prime contractor hired an unqualified mechanical subcontractor for this project. This mechanical contractor had primarily done residential work prior to this project. This contractor had no prior experience with VRF systems and only received the factory training to install these systems since they were awarded this project. Only the owner of the company actually received the training, and the rest of the employees that worked for this company did not receive it. As the owner was managing other projects, the owner could not pay adequate attention to the installation details for this project that had a large amount of refrigerant piping connections. The prime contractor also did not have an adequate QC staff to watch over the installation of the system. When it came time to attempt to pressure test all of the piping, the contractor had an extremely hard time finding any sections of pipe that would hold pressure. They were going through bottle after bottle of nitrogen attempting to track down and repair all of the leaks in the piping system. Many of these connections were flared connections. Overall due to the number of leaks and the lack of effort by the contractor to find the leaks on a routine basis, it took over two months to find all of the leaks. Now the staff that oversaw the installation of this system has a fear that leaks will be a problem in the future for this project as the contractor hired laborers to repair these leaks that were clearly not diligent in their efforts for the initial installation or the prolonged effort to repair the leaks. This case study further demonstrates the need for proper, thorough training for the installation of these systems and also reinforces the concerns in the US Army Corps of Engineers design guidance concerning the desired types of connections for VRF system being a soldered or brazed connection. 
Scott W. Kramer, et al. / Proceedings of the Creative Construction Conference (2019) 022 https://doi.org/10.3311/CCC2019-022

\section{Conclusion}

VRF systems offer a variety of advantages and disadvantages that must be weighed when considering their use. This level of consideration applies to all projects, not just the Corps of Engineers. Most of the HVAC industry experts interviewed felt the same way when asked if the Corps of Engineers should use these systems. The response was almost universally that the Government should consider their use for limited situations, but especially for renovation projects with limited ceiling space as they are one of few viable options in this scenario. However, based on the current design guidance and interviews with senior engineers, there are currently too many risks to universally start using these systems, and the bottom line is that they do not meet public law as explained above so they cannot be used for military projects. The unique training requirements that must be learned for each manufacturer is a major drawback. As more and more companies develop these systems, that problem will continue to grow. The limited amount of maintenance personnel at military installations cannot possibly remember the details for every manufacturer of these systems to be able to adequately maintain and replace them. An installation will need to have access to a service contractor that is certified for each vendor that will be installed on their base. So, while the systems contain great advantages, the risks that come with those advantages are heavy especially if a major problem occurs with the system and adequate training was not provided to service personnel as is unfortunately common for military projects.

A private owner that has more purchasing flexibility for parts and service has a little less risk than the Government does due to procurement regulations for not only materials, but labor and services as well. So, if a project team is considering the use of this type of system, it is highly recommended that all stakeholders thoroughly evaluate all the advantages and disadvantages that are applicable to their situation to ensure that the life of the project will be a success if a VRF system is going to be considered. All team members including the designer, contractor, building owner, maintenance staff, procurement staff, building occupants must be allowed to weigh in on their particular experiences and concerns with these systems to ensure that the VRF system is a proper fit for their business. To minimize the risks associated with these systems, a building owner must make sure that only individuals that are highly experienced, qualified and certified by the specific vendor to be used are allowed to design, install, and maintain the system to be used on their facility.

\section{References}

[1] Afify, R. (2008). Designing VRF Systems. ASHRAE Journal, 50(6), 52-55.

[2] Facts and Stats. (n.d.). Retrieved June 14, 2018, from https://www.energystar.gov/buildings/about-us/facts-and-stats

[3] Duda, S. (2012). Applying VRF? Don't overlook standard 15. ASHRAE Journal, 54(7), 18-24.

[4] US Department of Energy. (2008). Energy Efficiency Trends in Residential and Commercial Buildings. Energy Efficiency Trends in Residential and Commercial Buildings, 32.

[5] Woerpel, H. (2018a, February 5). HVACR Contractors Deem VRF Technology the Next Big Thing. Retrieved May 28, 2018, from https://www.achrnews.com/articles/136513-hvacr-contractors-deem-vrf-technology-the-next-big-thing

[6] Duggin, C. (2018, April 16). VRF: Overcoming challenges to achieve high efficiency. Retrieved May 27, 2018, from https://www.csemag.com/single-article/vrf-overcoming-challenges-to-achieve-high-efficiency/66488551d665c929f60fa54bbefe9d8b.html

[7] Carrier. (2018). Variable Refrigerant Flow.

[8] Daikin. (2018). VRV Product Catalog.

[9] LG. (2016). Multi V Engineering Manual.

[10] Mitsubishi. (2016). CitiMulti Catalog.

[11] ASHRAE. (2016). HVAC Systems and Equipment.

[12] Jankovic, A. (2016, September 27). Back to basics: VRF systems. Retrieved May 27, 2018, from https://www.csemag.com/singlearticle/back-to-basics-vrf-systems/3766b2f542fc80953d4d7ba5e5b2207b.html?tx_ttnews[sViewPointer]=1

[13] Inverter for Energy Saving | Benefits of Daikin Technology | Daikin Global. (n.d.). Retrieved June 30, 2018, from http://www.daikin.com/about/why_daikin/benefits/inverter/index.html 
Scott W. Kramer, et al. / Proceedings of the Creative Construction Conference (2019) 022 https://doi.org/10.3311/CCC2019-022

[14] Advantages of VRF | Carrier. (n.d.). Retrieved May 27, 2018, from https://www.carrier.com/commercial/en/us/vrf/advantages/

[15] Woerpel, H. (2018b, February 5). Knowledge is Power When It Comes to Variable Refrigerant Flow. Retrieved May 27, 2018, from https://www.achrnews.com/articles/136512-knowledge-is-power-when-it-comes-to-variable-refrigerant-flow

[16] Petrus, I., Hwang, R., \& McGee, D. (2017). Central Control Capabilities of VRF Systems. Engineered Systems, 34(8), 28-34.

[17] Goetzler, W. (2007). Variable Refrigerant Flow Systems. ASHRAE Journal, 49(4), $24+$. 